\title{
Evaluation of rapid screening methods in the diagnosis of urinary tract infection
}

\author{
Prakash N. ${ }^{1}$, Arundathi H. A. ${ }^{2}$, Halesh L. H. ${ }^{3}$, Siddesh K. C. ${ }^{4}$ \\ ${ }^{1,2}$ Assistant Professor, ${ }^{3}$ Professor \& HOD, ${ }^{4}$ Associate Professor, Dept. of Microbiology, ${ }^{1,3,4}$ Shimoga Institute of Medical \\ Sciences, Shimoga, Karnataka, ${ }^{2}$ Kanachur Institute of Medical Sciences, Deralakatte, Karnataka, India
}

*Corresponding Author:

Email: drarundathi88@gmail.com

\begin{abstract}
Introduction: Urinary Tract Infection (UTI) is one of the most common bacterial infection encountered in clinical practice. Even though urine culture is considered as the gold standard in detection of bacteriuria, many alternative screening methods like urine microscopy, chemical analysis using dipstick method and Gram's staining have been evaluated which are more economical and rapid. Hence, this study was undertaken to evaluate various screening methods in the rapid diagnosis of Urinary Tract Infections.

Objectives: To evaluate Gram staining of urine, leucocyte esterase and nitrite dipstick test methods in the rapid diagnosis of Urinary Tract Infections.

Materials and Methods: All urine samples received at Microbiology laboratory, McGann hospital, attached to Shimoga Institute of Medical Sciences, during study period was subjected to Gram staining, leucocyte esterase and nitrite dip stick test along with culture. Results of these tests were compared to culture results. Sensitivity and specificity of the tests was determined taking culture as standard.

Results: A total of 560 urine samples were processed. Culture showed significant bacteriuria in 210 cases. The sensitivity of urine microscopy was $70.9 \%$, leucocyte esterase test was $98 \%$ and nitrite test was $74.8 \%$. Whereas the specificity of urine microscopy was $70 \%$, leucocyte esterase test was $79.1 \%$ and nitrite test was $98.8 \%$. Similarly, the sensitivity of combined leucocyte esterase and nitrite tests was $90.9 \%$ and specifity was $97.4 \%$. Sensitivity and specificity of all the three screening tests combined were $95.7 \%$ and $94.8 \%$ respectively.

Conclusion: Screening tests such as, Urine microscopy by Gram's staining, Leucocyte esterase and nitrite dipstick test in combination can be used routinely to exclude bacteriuria. Urine culture can be employed only in the screening tests positive cases and in symptomatic cases irrespective of test results, thereby making diagnosis of UTI cost effective and rapid.
\end{abstract}

Keywords: Bacteriuria, Leucocyte esterase, Nitrite test, UTI, Urine microscopy.

\section{Introduction}

Urinary tract infections are one among the most common bacterial infections and account for a significant part of the workload in clinical microbiological laboratories. UTI can be either symptomatic or asymptomatic. Patients with significant bacteriuria who have symptoms referable to the urinary tract are said to have symptomatic bacteriuria whereas asymptomatic bacteriuria (ABU) is a condition characterized by bacteriuria without classical symptoms attributable to the urinary tract. ${ }^{1}$

Even though there are various methods for screening, urine culture is considered as the gold standard test for detecting bacteriuria. However, culture is expensive and takes $24-48 \mathrm{hr}$ to obtain results. To overcome these problems, many alternative screening methods have been evaluated, including urine microscopy, chemical analysis using a dipstick method, Gram staining, dipslide urine culture and bioluminescence. Each method has advantages and disadvantages in terms of sensitivity, specificity, adaptability, capital investment, running costs, automation and convenience for its use. ${ }^{2}$

Dipstick test, where detection of leucocyte esterase and nitrite is employed, is one of the qualitative diagnostic method used to detect UTI and have the advantage of being easy to perform, interpret, can be carried out in primary care giving facilities and result can be obtained immediately. Thus, this study was conducted to evaluate the diagnostic accuracy of the rapid dipstick test alone and in combination with Gram stain of uncentrifuged urine to predict urinary tract infection in comparison with the gold standard urine culture method. ${ }^{2}$

\section{Materials and Methods}

1. Source of data: The present study was conductedinMicrobiology laboratory, McGann hospital, attached to Shimoga Institute of Medical Sciences, Shimogafor a period of 6 months from July 2015 to December 2015. All urine samples were included in the study.

\section{Method of collection of data: Culture}

A semi-quantitative calibrated loop technique was adopted for the primary isolation of the organism. A loopful of well-mixed uncentrifuged urine was streaked on to the surface of Blood agar and Mac Conkey agar. After incubating aerobically for $24 \mathrm{~h}$ at $37^{\circ} \mathrm{C}$, colony forming unit (CFU) per millilitre of urine was calculated.Significant bacteriuria was taken as $\geq 10^{5} \mathrm{cfu} / \mathrm{ml}$. Samples showing growth of 2 or more bacteria were noted and repeat culture was asked for. The bacterial isolates in samples showing significant bacteriuria were identified by standard procedures and 
subjected to antibiotic susceptibility test according to CLSI guidelines. ${ }^{3-5}$

\section{Gram's staining of urine}

A drop of uncentrifuged well mixed urine was taken on a clean grease-free slide and stained by Gram's Method of staining and examined under the oil immersion objective of the microscope. Presence of $\geq 1$ bacteria per oil immersion field correlates with significant bacteriuria of $\geq 10^{5} \mathrm{cfu} / \mathrm{ml}$ of urine..$^{4,5}$

\section{Leukocyte esterase test and Nitrite test}

Uncentrifuged urine specimens were tested with colorimetric test for the presence of nitrite and leukocyte esterase activity, following the manufacturer's instructions. Briefly, the test strip was dipped in the urine sample and taken out immediately. The strips were blotted with a blotting paper to remove excess urine. Comparative reading was taken at 1 minute and 2 minute intervals for the LET and NT respectively.

For the purpose of the study, the urine culture was taken as the gold standard test. A positive result for an individual test that was also culture positive was taken as 'True positive'. Sensitivity and specificity for each test was calculated by using the formula:
Sensitivity $=\frac{\text { True positive }}{\text { All culture positive }} \times 100$
Specificity $=\frac{\text { True negative }}{\text { All culture negative }} \times 100$

Sensitivity and specificity of the two tests were combined in parallel to improve the diagnostic performance of the combined test. Combination in parallel means that the combined test result was interpreted as positive if any one test was positive, and interpreted as negative only when both the tests were negative.

\section{Results}

A total of 560 urine samples were received during the study period. Out of these, 210 samples showed significant bacteriuria by culture method. E.coli was the most prevalent organism isolated. Microscopy by Gram stain of uncentrifuged urine detected 149 cases of bacteriuria. Leucocyte esterase test was positive in 157 culture positive cases and Nitrite test in 166 culture positive tests.

Test results are as shown in table 1 and table 3 respectively. The sensitivity and specificity of individual tests and combined tests is shown in table 2 and table 4 respectively.

Table 1: Screening test results

\begin{tabular}{|l|c|c|c|}
\hline & Culture positive & Culture negative & Total \\
\hline $\begin{array}{l}\text { Urine } \\
\text { microscopy(gram } \\
\text { stain) }\end{array}$ & 149 & 07 & \\
\hline Positive & 61 & 343 & 156 \\
\hline Negative & 210 & 350 & 404 \\
\hline Total & & & 560 \\
\hline $\begin{array}{l}\text { Leucocyte } \\
\text { test }\end{array}$ & 157 & 105 & \\
\hline Positive & 53 & 245 & 262 \\
\hline Negative & 210 & 350 & 298 \\
\hline Total & 166 & & 560 \\
\hline Nitrite test & 44 & 346 & 170 \\
\hline Positive & 210 & 350 & 390 \\
\hline Negative & & & 560 \\
\hline Total & & & \\
\hline
\end{tabular}

Table 2: Sensitivity and Specificity of screening tests

\begin{tabular}{|l|c|c|}
\hline & Sensitivity (\%) & Specificity (\%) \\
\hline $\begin{array}{l}\text { Urine microscopy (Gram's } \\
\text { staining) }\end{array}$ & 70.9 & 98 \\
\hline Leucocyte esterase test & 74.8 & 70 \\
\hline Nitrite test & 79.1 & 98.8 \\
\hline
\end{tabular}

Table 3: Combined test results

\begin{tabular}{|l|l|l|l|}
\hline & Culture Positive & Culture Negative & Total \\
\hline $\begin{array}{l}\text { Leucocyte } \\
\text { esterase+nitrite test }\end{array}$ & & & \\
\hline Positive & 191 & 09 & 200 \\
\hline
\end{tabular}




\begin{tabular}{|l|l|l|l|}
\hline Negative & 19 & 341 & 360 \\
\hline Total & 210 & 350 & 560 \\
\hline $\begin{array}{l}\text { Leucocyte } \\
\text { esterase+nitrite+urine } \\
\text { microscopy }\end{array}$ & & & \\
\hline Positive & 201 & 18 & 219 \\
\hline Negative & 09 & 332 & 341 \\
\hline Total & 210 & 350 & 560 \\
\hline
\end{tabular}

Table 4: Sensitivity and Specificity of combined tests

\begin{tabular}{|l|c|c|}
\hline & Sensitivity (\%) & Specificity (\%) \\
\hline Leucocyte esterase + Nitrite test & 90.9 & 97.4 \\
\hline $\begin{array}{l}\text { Leucocyte esterase + Nitrite + } \\
\text { Urine microscopy }\end{array}$ & 95.7 & 94.8 \\
\hline
\end{tabular}

\section{Discussion}

Bacteriuria can be detected microscopically using Gram staining of uncentrifuged urine specimens, Gram staining of centrifuged specimens or by direct observation of bacteria in urine specimens. Gram stain of uncentrifuged urine specimens is a simple method.This test has the important advantage of providing immediate information as to the nature of the infecting organism and thereby guiding the physician in selecting empiric antimicrobial therapy. The important disadvantage of this test is, it is too labor- intensive., 4

By nitrite test, bacteriuria is detected chemically where bacteria produce nitrite from nitrate. The biochemical reaction that is detected by the nitrite test is associated with members of the family Enterobacteriaceae (pathogens most commonly responsible for UTIs), but the usefulness of the test is limited because nitrite production is not associated with urinary-tract pathogens such as S. saprophyticus, Pseudomonas species and Enterococci species. ${ }^{4,6}$

Leukocyte esterase tests are based on the hydrolysis of ester substrates by proteins with esterolytic activity. Human neutrophils produce as many as 10 proteins with esterolytic activity. These proteins react with ester substrates to produce alcohols and acids that then react with other chemicals to produce a colour change that is proportional to the amount of esterase in the specimen. A reagent strip impregnated with buffered in doxyl carboxylic acid ester and diazonium salt is used to detect leucocyte esterase. These tests have the advantage of detecting both esterases in intact leukocytes and esterases released after cell lysis. Therefore, even specimens that have not been preserved properly may yield a positive test result. False positive result may also be obtained from high levels of ascorbic acid and albumin in urine. ${ }^{4}$, 6

In our study, the sensitivity of urine microscopy, leucocyte esterase and nitrite test was $70.9 \%, 74.8 \%$ and $79.1 \%$ respectively. The specificity of these tests in the same order was $98 \%, 70 \%$ and $98.8 \%$ respectively. The sensitivity and specificity of combined leucocyte esterase and nitrite tests was $90.9 \%$ and $97.4 \%$ respectively. Sensitivity and specificity of all the three screening tests combined was $95.7 \%$ and $94.8 \%$ respectively

Other studies which have evaluated the efficacy of these screening tests are, study by Nayak et al, who have reported the sensitivity and positive predictive value of dipstick test as $68 \%$ and $71.4 \%$

respectively. ${ }^{7}$ Jayalakshmi et al who have concluded that dipstick test with both Leucocyte esterase and nitrite detection has advantage as screening test with sensitivity of $82.9 \%{ }^{8}$

Our test results confirm and expand the findings of Titoria $\mathrm{A}$ et $\mathrm{al}^{1}$ and Taneja $\mathrm{N}$ et $\mathrm{al}^{9}$ who have reported almost the same diagnostic performance of these screening tests and have concluded that these tests when combined are of great advantage in screening urinary tract infection.

Therefore, even though urine culture is the gold standard for the diagnosis of urinary tract infection, due to cost and delay of result in culture, combined dipstick with urine microscopy by Gram's staining, which is rapid and cheaper can be used as the initial step in UTI diagnosis. A positive screening test will require culture and sensitivity testing so that the right antibiotic can be prescribed. This will decrease a huge work burden in the laboratory as well as cost burden on the patients, like 332 culture negative cases which were detected by combined screening tests itself in the present study. There will be few cases which could be missed by screening tests, like 9 out of 210 cases in the present study. This can be tackled by doing culture in symptomatic cases even with negative screening results.

\section{Conclusion}

Use of combined screening tests like urine microscopy by Gram's staining, leucocyte esterase test and Nitrite test as a routine laboratory practice for faster diagnosis of UTI, where culture is performed only when combined screening tests are positive or in 
symptomatic patients even with negative results appears rational and cost effective.

\section{References}

1. Demilie T, Beyene G, Melaku S, Tsegaye W. Diagnostic accuracy of rapid urine dipstick test to predict urinary tract infection among pregnant women in Felege Hiwot Referral Hospital, Bahir Dar, North West Ethiopia. BMC Research Notes 2014;7:481.

2. Titoria A, Gupta A, Rathore M, Prakash SK, Rawat D, Manaktala U. Asymptomatic bacteriuria in women attending an antenatal clinic at a tertiary care centre. SAJOG. 2014;20(1):4-7.

3. Clinical and Laboratory Standard Institute. Performance Standard for Antimicrobial Susceptibility Testing. Twenty fifth Informational Supplement. M 100-S 26.Wanye, PA, USA. CLSI 2015.

4. Winn W, Allen S, Janda W, Koneman E, Procop G, Schreckenberger et al. Guidelines for the collection, transport, processing, analysis, and reporting of cultures from various specific specimen sources. In. "Koneman's colour Atlas and Textbook of Diagnostic Microbiology". $6^{\text {th }}$ ed. Philadelphia: Lippincott Williams and Wilkins publishers. 2006;82-7.

5. Colee JG, Fraser AG, Marmion BP, Simmons A. Mackie and McCartney Practical Medical Microbiology. $14^{\text {th }}$ ed. New York: Elsevier Publishers; 1999.

6. Wilson ML, Gaido L. Laboratory diagnosis of urinary tract infections in adult patients. Medical Microbiology. CID. 2004;38;1150-7.

7. Nayak US, Solanki, Patva P. Utility of dipstick versus urine culture in diagnosis of urinary tract infection in children. Gujarat Medical Journal. 2010;65(1):20-2.

8. Jayalakshmi J, Jayaram VS. Evaluation of various screening tests to detect Asymptomatic bacteriuria in pregnant women.Indian Journal of Pathology and Microbiology. 2008;51(3):379-81.

9. Taneja N, Chatterjee SS, Singh M, Sivapriya S, Sharma M, Sharma SK. Validity of Quantitative Unspun Urine Microscopy, Dipstick Test Leucocyte Esterase and Nitrite Tests in Rapidly Diagnosing Urinary Tract Infections. JAPI. 2010;58:485-7.

How to cite this article: Prakash N, Arundathi HA, Halesh LH, Siddesh KC. Evaluation of rapid screening methods in the diagnosis of urinary tract infection. Indian J Microbiol Res 2018;5(1):20-23. 